\title{
Detection of Ceramic Cracks Using a Distributed High-Resolution Brillouin Fiber Optic Sensor
}

\begin{abstract}
Maria Feng ${ }^{*}$, Lufan Zou ${ }^{* *}$, and Michio IMaI*
Abstract : A distributed sensor system is highly desirable for detecting, locating, and monitoring fine cracks at unknown locations in advanced ceramics. This paper presents a distributed high-resolution fiber optic sensor based on the Brillouin scattering principle, and its application in ceramic crack detection for the first time. The existence of cracks, together with their locations, is identified by measuring the strain distribution on a sensing fiber bonded to the ceramic surface. By employing the innovative coherent probe-pump interaction technique, the Brillouin sensor developed in this study achieves a high spatial resolution $(100 \mathrm{~mm})$ and measurement accuracy. Capable of detecting and locating fine cracks less than $40 \mu \mathrm{m}$, the efficacy of the distributed Brillouin fiber optic sensor is demonstrated through experiments.
\end{abstract}

Key Words : Brillouin scattering, crack detection, strain measurement, fiber optic sensor, advanced ceramics.

\section{Introduction}

Advanced ceramics are valued for their hardness, hightemperature strength, light weight and abrasion resistance, and hence find wide use in such applications as ballistic protective armor components, wear components of equipment in oil, gas, and mining operations, and high-performance engines. However, due to the brittle nature, advanced ceramics are subjected to cracking upon impact during operation. Cracks, including invisible micro cracks, significantly degrade the strength of a ceramic component.

The traditional inspection of cracks in ceramics relies on visual inspection including the dye and the fluorescent penetrant methods. Recently nondestructive evaluation (NDE) methods such as X-ray and ultrasonic imaging have been applied to detect cracks in ceramics, but have limited sensitivities to micro cracks. Both the visual and NDE inspection methods are laborious, time consuming, and more importantly, difficult to perform continuously during operation.

Optical fiber based sensing has received increasing attention over the last two decades for the purposes of structural health monitoring. Different sensing techniques have been developed to monitor specific parameters. Recently it has been reported that fiber Bragg grating (FBG) sensors were applied to detect cracks in carbon fiber reinforced polymer composites with a strain resolution of $1 \times 10^{-6}$ [1]. The fiber optic sensor technology can be embedded in or surfaced-bonded to the material for continuous monitoring. A typical FBG sensor, however, has a limited gage length and thus works as a "point" sensor [2]. Although the sensing gages can be multiplexed, the number of multiplexible gages is limited. Since the crack locations in a material are not known a-priori, conventional "point" sensors, even if they can be multiplexed, are not effective in crack detection. A truly distributed sensor system is desired for detection and continuous monitoring of cracks. Recently a study on

\footnotetext{
${ }^{*}$ The University of California, Irvine, CA, USA

** OZ Optics, Ltd., K0A1L0, Canada

(Received September 24, 2009)

(Revised February 24, 2010)
}

crack detection using a distributed Brillouin fiber optic sensor was reported, which successfully detected a crack with a gap of $0.5 \mathrm{~mm}$ by taking advantage of the delamination over a $10 \mathrm{~cm}$ length between the specialty optical fiber sensor cable and the structure around the crack [3].

This paper presents a distributed Brillouin fiber optic sensor with a higher spatial resolution $(100 \mathrm{~mm})$, capable of detecting a fine crack with a gap in the order of $10 \mu \mathrm{m}$. In this study, the capability of the sensor in detecting and locating micro cracks is quantitatively demonstrated using a micro crack (induced on a PVC plate) with a controllable crack width. Furthermore, optical fibers are bonded on the surface of ceramic tiles in different layout patterns to detect cracks caused by impact.

\section{Sensing Principle of Distributed Fiber Optic Sen- sor}

The distributed strain sensing capability of the Brillouin fiber optic sensor provides a potential tool for detecting cracks at unknown locations in materials and structures by measuring a strain profile along the entire length of a sensing fiber, rather than strains at discrete points. One class of the distributed Brillouin fiber optic sensor is based on the Brillouin loss technique [4], whereby two counter-propagating laser beams, a pulsed Stokes beam and a continuous wave (cw) pump beam, exchange energy through an induced acoustic field. The interaction magnifies the pulsed Stokes beam at the expense of depleting the pump beam, which is then detected as a loss signal. The maximum depletion of the pump beam at a point along the fiber occurs when the frequency of the acoustic wave $v_{B}$ at that point matches the beat frequency of two laser beams, i.e., $v_{p}-v_{s}=v_{B}$, where $v_{p}$ and $v_{s}$ are the frequencies of the pump and Stokes beams, respectively. The frequency of the acoustic wave, hereafter referred to as the Brillouin frequency shift, is related to the fiber properties and the laser wavelength. The sensing capability of Brillouin scattering arises from the dependence of the Brillouin frequency shift, $v_{B}$, on the local acoustic velocity and refractive index in the fiber core glass, which has a linear temperature and strain dependence through 


$$
\begin{aligned}
& v_{B}\left(T_{0}, \varepsilon\right)=C_{\varepsilon}\left(\varepsilon-\varepsilon_{0}\right)+v_{B 0}\left(T_{0}, \varepsilon_{0}\right) \\
& v_{B}\left(T, \varepsilon_{0}\right)=C_{T}\left(T-T_{0}\right)+v_{B 0}\left(T_{0}, \varepsilon_{0}\right)
\end{aligned}
$$

where $C_{\varepsilon}$ and $C_{\mathrm{T}}$ are the strain and temperature coefficients, and $\varepsilon_{0}$ and $T_{0}$ are the strain and temperature corresponding to a reference Brillouin frequency $v_{B 0}$ [5],[6]. By scanning the beat frequency of the two lasers and measuring the intensity of the corresponding signal, a Brillouin loss spectrum is obtained. As a result, continuous temperature and strain distributions can be obtained.

The distributed Brillouin loss sensor can measure, in principle, the strain and temperature at any point along the sensing fiber. Spatial information along the fiber length is obtained through the Brillouin Optical Time Domain Analysis (BOTDA) by measuring the propagation time for the light pulses to travel in the fiber. The spatial resolution is determined by $\delta z=c W /(2 n)$ [7], where $c, n$, and $W$ are the speed of light in vacuum, the refractive index of fiber core, and the Stokes pulse width, respectively. The spatial resolution can be adjusted for different applications simply by altering the pulse duration even after the sensing fiber is installed, making this type of Brillouin sensor highly suitable for structural health monitoring.

\section{High-Resolution and High-Accuracy Brillouin Sen- sor}

Detection of fine cracks in materials and structures requires a high spatial resolution. A Brillouin-scattering-based sensor system with a $100 \mathrm{~mm}$ spatial resolution was developed for this study. A combination of continuous wave (cw) and pulse source as the probe (Stokes) beam interacts with the cw laser as the pump beam in the probe-pump Brillouin sensor system [8]. The Brillouin interaction of the Stokes and the pump beams in the fiber includes both the DC-pump and the pulse-pump interactions. The DC component can be separated into two portions by their phases after propagating through the electro-optic modulator (EOM).

$E_{\text {out }}= \begin{cases}A_{\mathrm{DC}} \cos \left(\omega t+\varphi_{\mathrm{DC}}\right) & t<t_{0}-\frac{\tau_{p}}{2} \text { and } t>t_{0}+\frac{\tau_{p}}{2} \\ A_{\text {pulse }} \cos \left(\omega t+\varphi_{\text {pulse }}\right) & t_{0}-\frac{\tau_{p}}{2} \leq t \leq t_{0}+\frac{\tau_{p}}{2}\end{cases}$

where $A_{\mathrm{DC}}$ is the electrical amplitude of the DC component. As shown in Fig. 1, the electrical amplitude of the pulsed portion, $A_{\text {pulse }}$, can be divided into two parts: $a_{\mathrm{DC}}$, the same amplitude as the DC component $A_{\mathrm{DC}}$, and $a_{\text {pulse }}$, a super Gaussian pulse centered at $t_{0}$ with a width of $\tau_{p}$, and yields

$$
E_{\text {out }}= \begin{cases}A_{\mathrm{DC}} \cos \left(\omega t+\varphi_{\mathrm{DC}}\right) & t<t_{0}-\frac{\tau_{p}}{2} \\ a_{\mathrm{DC}} \cos \left(\omega t+\varphi_{\text {pulse }}\right) & \text { and } t>t \\ +a_{\text {pulse }} \cos \left(\omega t+\varphi_{\text {pulse }}\right) & t_{0}-\frac{\tau_{p}}{2} \leq t \leq t_{0}+\frac{\tau_{p}}{2}\end{cases}
$$

or

$$
E_{\text {out }}=\left\{\begin{array}{l}
A_{\mathrm{DC}} \cos \left(\omega t+\varphi_{\mathrm{DC}}\right)+a_{\mathrm{DC}} \cos \left(\omega t+\varphi_{\text {pulse }}\right) \\
a_{\text {pulse }} \cos \left(\omega t+\varphi_{\text {pulse }}\right)
\end{array}\right.
$$

Consequently, the Brillouin interaction of the pump and Stokes (i.e. $E_{\text {out }}$ ) in the fiber consists of both pump-DC and pump-pulse interactions. Note that the Brillouin loss spectrum produced by the interaction of the first DC part (Eq. 4a) with the pump has only information of strain and/or temperature along the sensing fiber, but no information about locations. Whereas

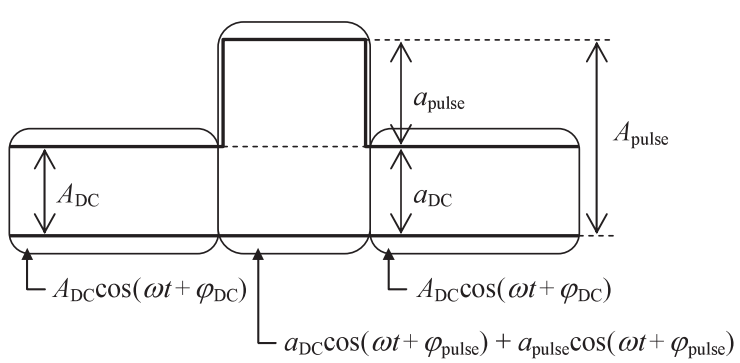

or

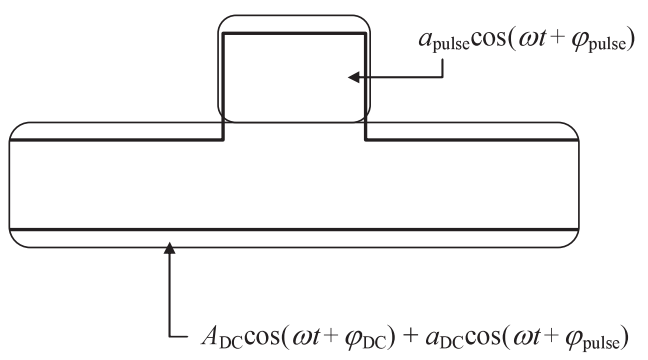

Fig. 1 Schematic diagram of DC and pulse components.

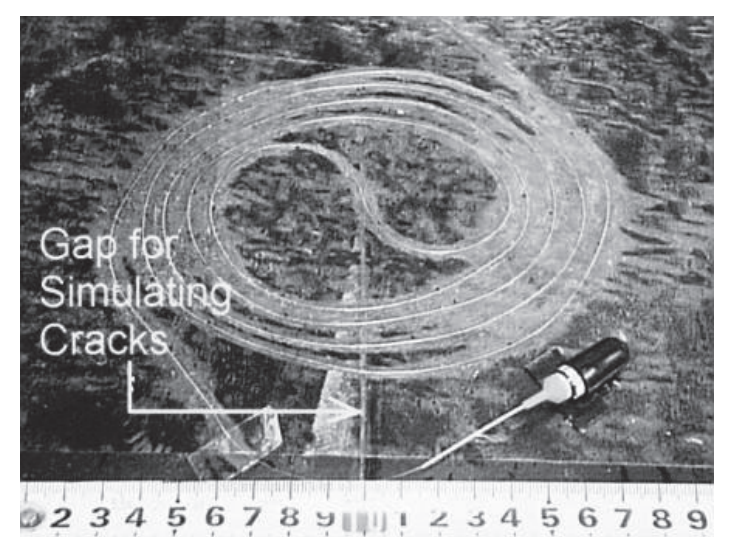

Fig. 2 Detection of simulated cracks.

the interaction of the pump with the DC component inside the phase-length (the second DC part of Eq. 4a) is coherent with the pulse-pump interaction, it amplifies the Brillouin signal significantly. This provides localized information of strain and/or temperature and enhances measurement accuracy.

Based on the unique coherent probe-pump interaction technique, a Brillouin sensor system was fabricated and its high resolution and high accuracy were demonstrated by an experiment, in which cracks with controllable widths were detected. Figure 2 shows the experimental setup. A PVC plate was used in this setup due to the ease in initiating a micro crack with a controllable width. As shown in the figure, a small gap was first created in the PVC plate. Then the sensing fiber was bonded on the plate in a double spiral shape, passing through the gap four times. A strain distribution (profile) along the entire sensing fiber was measured as a pre-cracking baseline. After the fiber was bonded, the gap width was increased by $40 \mu \mathrm{m}$ at the point where the most outside loop of the sensing fiber was located, to create simulated cracks at the four fiber locations. The strain distribution was measured again and the measurement normalized by the pre-cracking baseline is shown in Fig. 3. The four strain peaks corresponds well to the four locations of sensing fiber passing through the gap. 


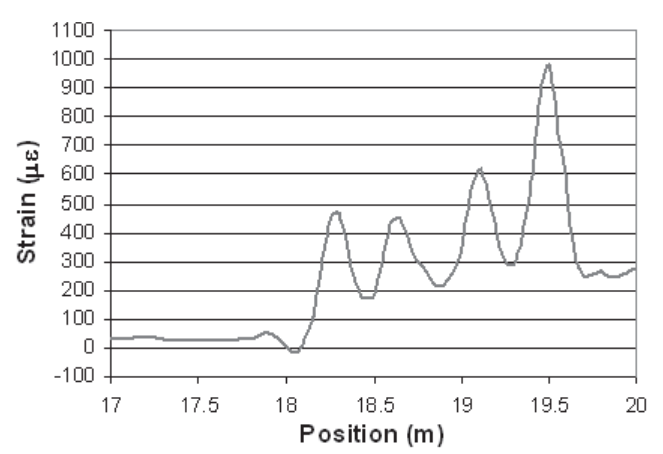

Fig. 3 Strain distribution along the sensing fiber.

The Brillouin sensor system successfully detected four simulated fine cracks at different locations of the fiber. The largest crack width was $40 \mu \mathrm{m}$, located on the most outside loop of the fiber and the crack width decreased toward the center of the fiber loops. The smallest distance between two adjacent cracks was approximately $300 \mathrm{~mm}$. The locations of the four strain peaks were consistent with the actual crack locations. This experiment demonstrated the high performance of the developed Brillouin sensor system in terms of the spatial resolution, sensitivity and accuracy.

\section{Crack Detection Tests}

The high spatial resolution of the distributed Brillouin sensor system developed in this study is ideal for detecting fine cracks, as the locations of the cracks are usually unknown. Experiments were further performed to evaluate the capability of the Brillouin sensor for detecting and locating fine cracks in advanced ceramics. These materials have wide industrial and defense applications, but they are subjected to cracking upon impact and such cracks can significantly degrade the material performance. Often these cracks are invisible due to the size of the cracks, covers on the ceramics or their locations difficult to access. Currently there is no cost-effective method to detect and evaluate such cracks. In this study, we used the Brillouin fiber optic sensor system developed in this study for detecting and locating cracks on ceramics.

\subsection{Test Specimen and Procedure}

Ceramic tile specimens with a dimension of $250 \times 300 \times$ $8 \mathrm{~mm}$ were prepared. An acrylate buffered SMF-28 optical fiber was used as the sensing fiber, which was bonded on the surface of the ceramic specimens. There were two technical challenges when bonding the fiber on the ceramic tile. First, it is crucial that the fiber stretches accordingly as cracks occurs on the ceramic surface, without being affected by the elasticity of the bond. Second, the fiber should be bonded in such a layout pattern that the entire surface of the tile is covered. A number of bonding materials were studied, based on which a brittle bond with a short (less than 1 minute) curing time, a cyanoacrylatebased adhesive, was selected. A number of fiber layout patterns were investigated. Figures 4 (a) and (b) show two of the ceramic specimens, Specimens I and II, with different fiber layout patterns. Specimen I is bonded with a fiber laid in straight parallel lines. Twelve sensing segments of the fiber were bonded externally on the ceramic surface. To avoid sharp bending of optical fiber, a $500 \mathrm{~mm}$ loose fiber segment was provided between two adjacent bonding segments, each $200 \mathrm{~mm}$ long. In Specimen

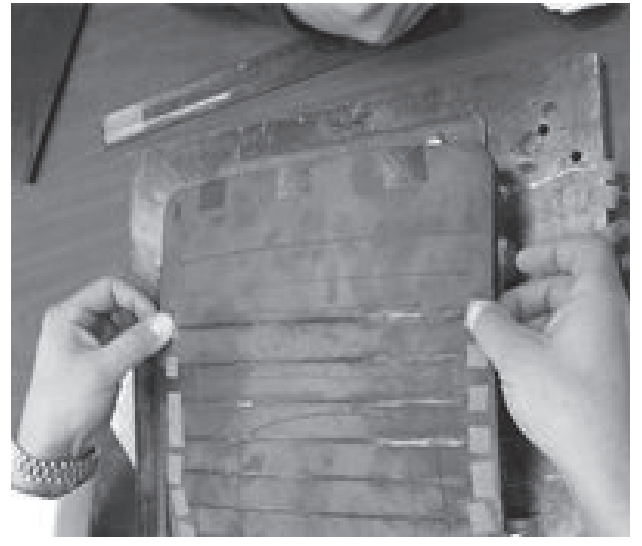

(a) Specimen I.

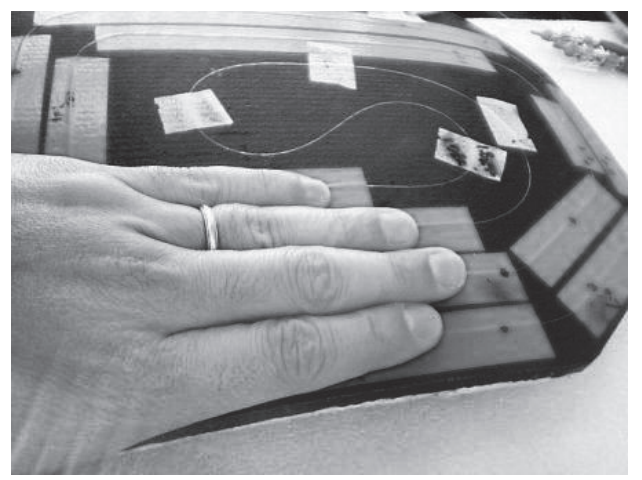

(b) Specimen II.

Fig. 4 Ceramic tiles with different sensor layout patterns.

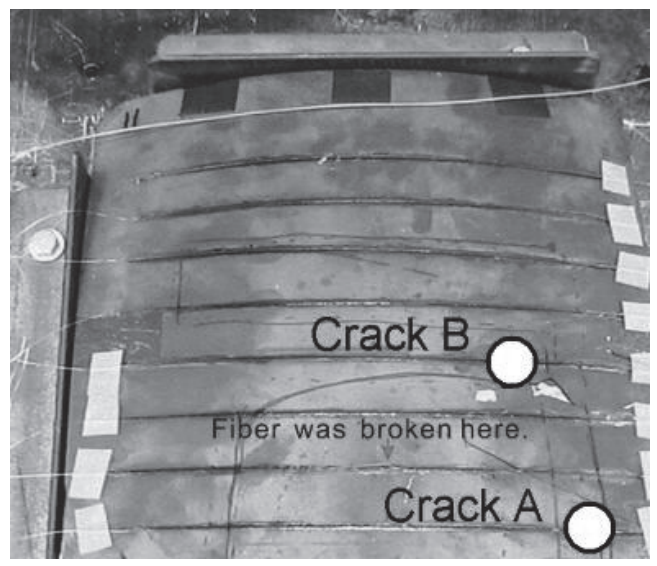

Fig. 5 Cracks A and B running through the sensing fiber.

II, a sensing fiber is bonded in a double spiral pattern.

The Brillouin measurement was taken on the ceramic tile before cracking as the baseline measurement. Then fine cracks were induced to the ceramic tile by subjecting the tile to compressive loading or dropping onto a concrete surface. The sensor measurement was taken again to detect the ceramic cracks by analyzing the Brillouin loss spectrum.

\subsection{Results and Discussion}

The cracked Specimen I is shown in Fig. 5, in which two cracks, labeled as A and B, ran through the sensing fiber. Measurement was taken again after the specimen cracked.

Figure 6 displays the Brillouin loss spectra of the optical sensing fiber at the room temperature before the ceramic tile 


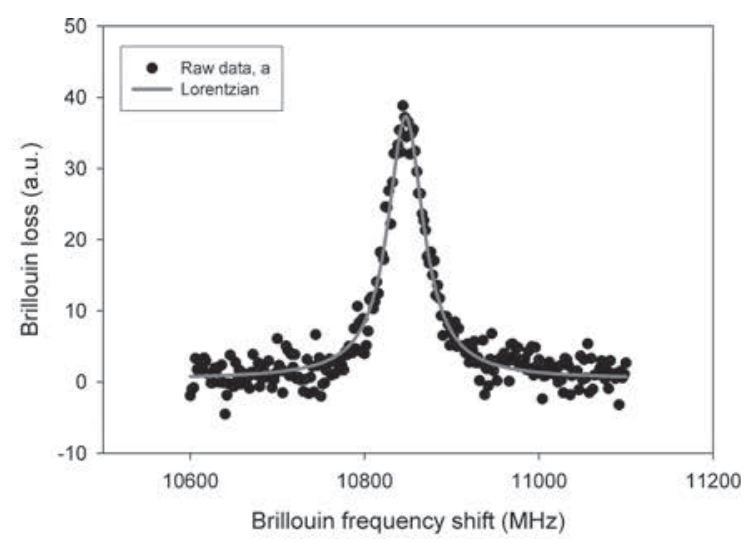

(a) Sensing fiber free of strain.

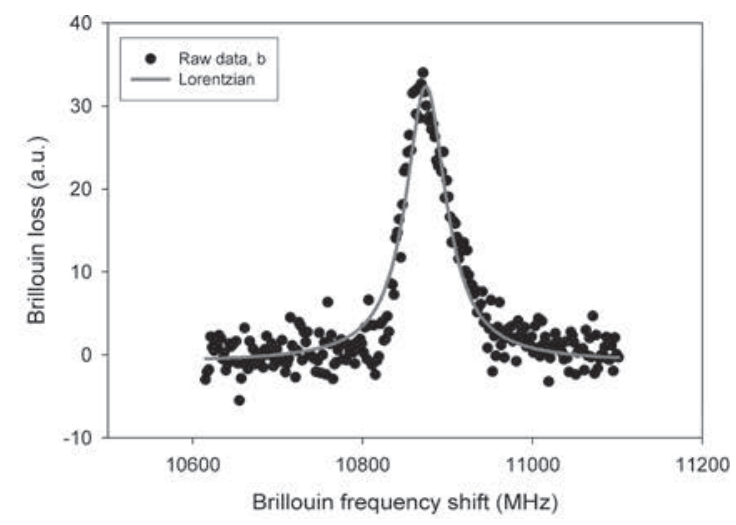

(b) Pre-strained sensing fiber.

Fig. 6 Brillouin loss spectrum of the sensing fiber on un-cracked ceramic tile.

was subjected to cracking, in which the central Brillouin frequency was measured as $10,847.10 \mathrm{MHz}$ with a line-width of 49.14 MHz (Fig. 6(a)) with free of strain of sensing fibers and $10,874.47 \mathrm{MHz}$ with a line-width of 49.72 MHz measured from pre-strained sensing fibers on the ceramic tile (Fig. 6 (b)). Compared Fig. 6(b) to Fig. 6 (a), the spectrum measured from strained sensing fiber can not be accurately modeled by a Lorentzian function, because the strain distribution causes Brillouin spectra to be deviated from a standard Lorentzian shape. In the experiment, the pulse width was $1 \mathrm{~ns}$ with extinction ratio of $15 \mathrm{~dB}$ and the line-width of the Brillouin spectrum was around $50 \mathrm{MHz}$ that would assure to obtain an accurate measurement of the strain. It is noted the Brillouin fiber optic sensor is a direct measurement of the Brillouin frequency shift, and the strain/temperature are computed according to Eqs. (1) and (2). Therefore, to obtain a narrow Brillouin spectrum that is close to the intrinsic Brillouin line-width $35 \mathrm{MHz}$ of the pure cubic silica is a fundamental requirement for accurate measurement. The measurement was taken as the strain baseline from the un-cracked ceramic tile.

After the ceramic tile was subjected to the compressive loading test, creating cracks A and B running through the sensing fiber as shown in Fig. 5, the strain distribution along the fiber was measured again. Since the sensing fiber bonded on the middle of the ceramic tile was damaged by direct contact with attachment of universal testing machine during the compressive loading test, the fiber was spliced and two separate measurements were taken that covered Crack A and Crack B, respectively. The Brillouin loss spectra measured at the two

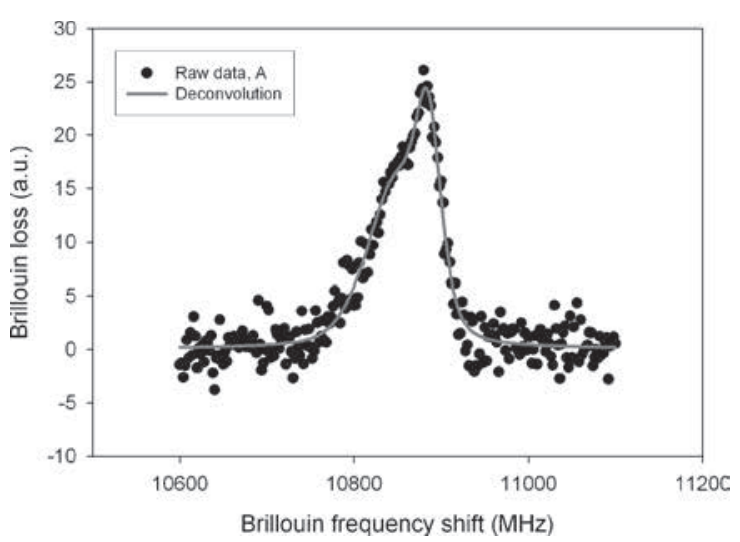

Fig. 7 Brillouin loss spectrum at Crack A.

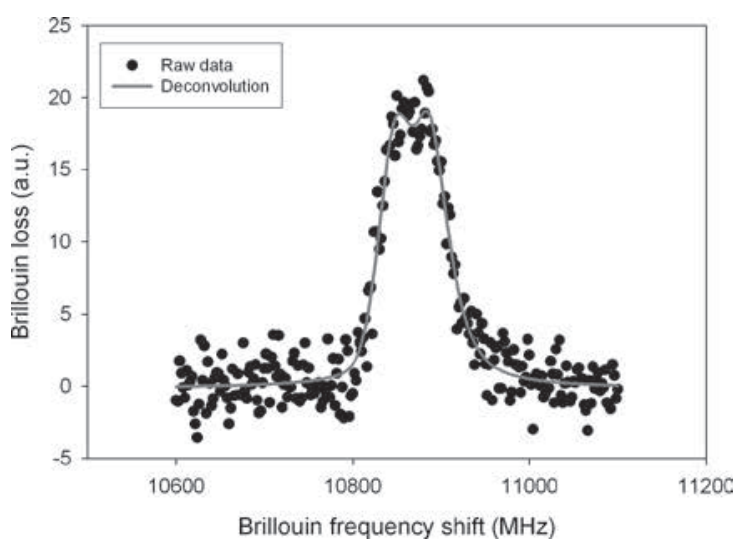

Fig. 8 Brillouin loss spectrum at Crack B.

crack locations are displayed in Figs. 7 and 8, in which the Brillouin central frequencies at the fiber locations of Crack A and Crack B are 10,884.83 MHz and 10,886.84 MHz, respectively. The Brillouin spectra are asymmetric, because (1) each of the $200 \mathrm{~mm}$ stretched (bonded) sensing fiber segment was separated by a $500 \mathrm{~mm}$ loose fiber segment to avoid additional optical loss by bending (see Fig. 4 (a)), (2) the crack caused a non-uniform strain distribution along the fiber, (3) the sampling resolution is $50 \mathrm{~mm}$, and (4) the spatial resolution is $100 \mathrm{~mm}$. The Brillouin interaction of the probe (Stokes) and the pump beams in the fiber includes both the DC-pump and the pulsepump interactions. Thus the Brillouin spectrum is the convolution of both the DC-pump and the pulse-pump interactions. Since the DC-pump interaction provides overall strain information along the whole sensing fiber and the pulse-pump gives local strain information, we have to deconvolute the Brillouin spectrum to obtain Brillouin frequencies on the crack locations. The solid curve represents the deconvolution of the Brillouin frequencies based on the coherent interaction of the probe and pump beams [8].

Normally the spatial resolution can be increased by narrowing the input pulse in time domain. However, the narrow pulse will produce a wide Brillouin spectrum in frequency domain, which will reduce the resolution and accuracy of strain and temperature measurement since Brillouin sensor is indirect measurement of strain and temperature according to the calculation from Eqs. (1) and (2). Due to the use of the coherent technology of probe-pump interaction in the Brillouin sensor, the Brillouin spectra, as shown in Figs. 6, 7, and 8, display less than $50 \mathrm{MHz}$ line-width that assure to achieve an accurate measurement of 


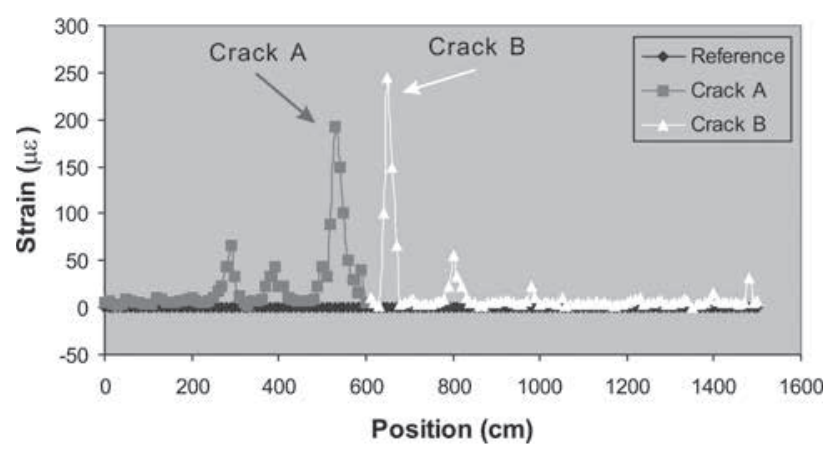

Fig. 9 Strain distribution on cracked ceramic tile.

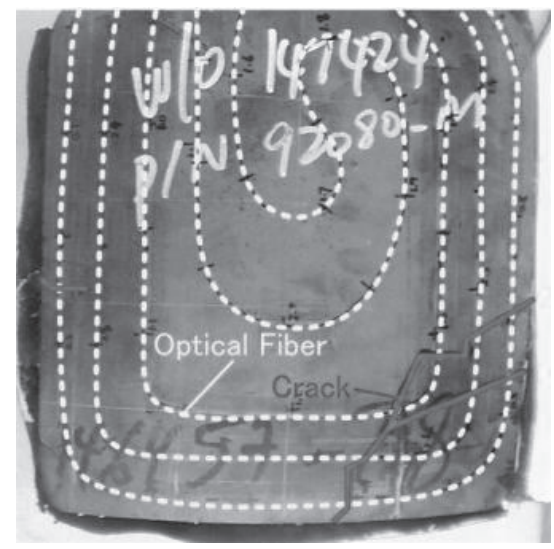

(a) Specimen II after cracking.

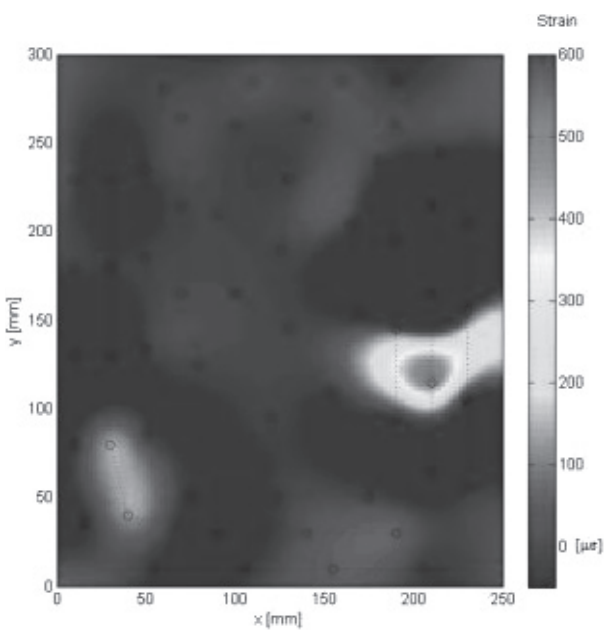

(b) Strain contour.

Fig. 10 Test of Specimen II.

the strain.

The strain distributions along the sensing fiber are shown in Fig. 9, which includes the 1st measurement (before cracking) as the baseline reference, the 2nd measurement (after cracking) to identify Crack A, and the 3rd measurement (after cracking) to find Crack B. The two distinctive peaks of the strain with strain values of $(196 \pm 2) \times 10^{-6}$ and $(249 \pm 2) \times 10^{-6}$ are corresponding to Crack A and Crack B, respectively. Even though there are some ripples along the strain distribution, no crack was identified. Thus, it is assumed that such ripples are associated with small changes in strains at certain fiber locations. Further study is required to determine the minimum detectable crack. This experiment successfully demonstrated the capabil- ity of the developed distributed Brillouin fiber optic sensor system in detecting fine cracks on ceramics.

The cracked Specimen II is shown in Fig. 10 (a). Same measurements as those discussed above for Specimen I were taken before and after cracks occurred. Based on the measured strain profile along the sensing fiber, a strain contour is plotted as shown in Fig. 10 (b). Crack locations are not perfectly identified due to the limitation in the sensor's spatial accuracy. Nevertheless the highest strain areas correspond reasonably well with visually observed cracks.

It is observed that both the straight and the double spiral layout patterns tested in this study are effective in mapping a surface of a ceramic tile for crack detection. Attention needs to be paid to the curvature of the sensing fiber when laying in the spiral pattern to prevent the loss of optical power.

It is noted that the sensor measurements were taken while keeping the room temperature constant in this study. The same Brillouin sensor system can be used for measuring temperature distribution along a sensing fiber. Techniques are being developed to simultaneously measure and distinguish strain and temperature, but they are beyond the scope of this paper.

\section{Conclusion}

This paper has presented the advancement of the Brillouin fiber optic sensing technology and the first effort in applying this technology for detecting micro cracks in ceramics. By employing the unique coherent probe-pump interaction technique, a distributed high-performance Brillouin strain sensor system was developed with a $100 \mathrm{~mm}$ spatial resolution, a significant advancement over the conventional resolution of $1 \mathrm{~m}$. By measuring frequency shift of the Brillouin loss spectra before and after cracks, the sensor system successfully detected the change of the strain profile along the sensing fiber associated with cracks (with a width less than $40 \mu \mathrm{m}$ ). Not only the existences of the cracks were detected, their locations were also successfully identified. From a practical application point of view, the study also proved that both the straight and the spiral fiber layout patterns were effective in mapping a surface of a ceramic tile for detecting cracks at unknown locations.

\section{References}

[1] Y. Okabe, N. Tanaka, and N. Takeda: Effect of fiber coating on crack detection in carbon fiber reinforced plastic composites using fiber Bragg grating sensors, Smart Materials and Structures, Vol. 11, pp. 892-898, 2002.

[2] W.L. Schulz, J.P. Conte, and E. Udd: Long-gage fiber optic Bragg grating strain sensors to monitor civil structures, Proceedings of SPIE, Vol. 4330, pp. 56-65, 2001.

[3] F. Ravet, F. Briffod, B. Glisic, M. Nikles, and D. Inaudi: Detection of sub-millimeter faults with a time domain distributed Brillouin sensor, Proceedings of SPIE, Vol. 7004, 700412, 2008.

[4] L. Zou, G.A. Ferrier, S.V. Afshar, Q. Yu, L. Chen, and X. Bao: Distributed Brillouin scattering sensor for discrimination of wall-thinning defects in steel pipe under internal pressure, $A p$ plied Optics, Vol. 43, No. 7, pp. 1583-1588, 2004.

[5] T. Horiguchi, T. Kurashima, and M. Tateda: Tensile strain dependence of Brillouin frequency shift in silica optical fibers, IEEE Photonics Technology Letters, Vol. 1, No. 5, pp. 107-108, 1989.

[6] T. Kurashima, T. Horiguchi, and M. Tateda: Thermal effects on the Brillouin frequency shift in jacketed optical silica fibers, Applied Optics, Vol. 29, No. 15, pp. 2219-2222, 1990.

[7] L. Zou, X. Bao, S.V. Afshar, and L. Chen: Dependence of the 
Brillouin frequency shift on strain and temperature in a photonic crystal fiber, Optics Letters, Vol. 29, No. 13, pp. 1485-1487, 2004.

[8] L. Zou, X. Bao, Y. Wan, and L. Chen: Coherent probe-pumpbased Brillouin sensor for centimeter-crack detection, Optics Letters, Vol. 30, No. 4, pp. 370-372, 2005.

\section{Maria Feng}

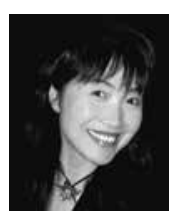

She is a Professor of Civil and Environmental Engineering and Endowed Chair in the Center for Diversity in Engineering Education at the University of California, Irvine (UCI). She is the Founding Director of the Center for Advanced Monitoring and Damage Inspection at UCI. With a Ph.D. in Mechanical Engineering from the University of Tokyo, she has a wide variety of research interests focusing on the interdisciplinary field of infrastructure monitoring through development and application of sensor technologies. She has received the NSF Career Award, the ASCE/ASME/IEEE Alfred Noble Prize, the ASCE Collingwood Prize, the ASCE Huber Prize, and she has been elected Fellow of ASCE.

\section{Lufan Zou}

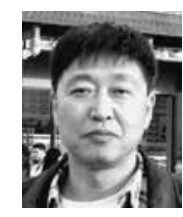

He received the Ph.D. degree in semiconductor physics and device from the Institute of Semiconductor, Chinese Academy of Science, Beijing, China, in 1997. He is a Technical Manager in OZ Optics, Ltd., Ottawa, ON, Canada. He, as the first author, and co-authors presented theory and experiments of coherent probe-pump-based Brillouin scattering to achieve centimeter spatial resolution with high-frequency resolution in the article of "Coherent probe-pump based Brillouin sensor for centimeter-crack detection," which has been published in Optics Letters, Vol. 30, No. 4, pp. 370-372, Feb. 2005.

\section{Michio ImaI}

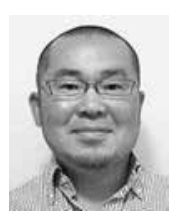

He received his B.E. degree from Keio University, Japan, in 1995, and M.S. and Ph.D. degrees from the University of California at Irvine, USA, in 2007 and 2009, respectively. He is currently working in Kajima Technical Research Institute, Japan, and engaged in research on structural health monitoring using fiber optic sensor. He is a member of SPIE and JSCE. 